\title{
BALANÇO HÍDRICO EM SOLO COM CULTIVOS DE SUBSISTÊNCIA NO SEMI-ÁRIDO DO NORDESTE DO BRASIL
}

\author{
Antonio Celso Dantas Antonino ${ }^{1}$, Everardo V. S. B. Sampaio ${ }^{1}$, Attilio Dall'Olioº \& \\ Ignácio Hernan Salcedo ${ }^{1}$
}

\begin{abstract}
RESUMO
Tendo em vista ser a água limitante e haver poucos estudos sobre o balanço hídrico em cultivos do semi-árido nordestino, instalou-se um experimento em Coxixola, $\mathrm{PB}$, com quatro tratamentos (plantios de milho e de feijão, solo nu e com cobertura morta), parcelas de 7,7 x $10 \mathrm{~m}$ e espaçamento de plantio de 1,1 x 1,0 m. Foram medidos chuva, evaporação (Tanque "classe A") e armazenamento de água no solo (sonda de nêutrons). A chuva pouca $(212 \mathrm{~mm}$ ) e mal distribuída causou restrição hídrica na parte final do experimento e foi responsável pelas baixas eficiências de uso de água e baixas produtividades do milho (grãos e biomassa total, 282 e $1141 \mathrm{~kg} \mathrm{ha}^{-1}$ ) e do feijão $\left(166 \mathrm{e} 558 \mathrm{~kg} \mathrm{ha}^{-1}\right)$. Solo nu e com cobertura morta tiveram comportamento semelhante, com grandes perdas de água (1,57 e 1,48 $\mathrm{mm} \mathrm{dia}^{-1}$, respectivamente) e apenas pequenos aumentos nas lâminas armazenadas no perfil do solo, ao final do experimento ( $28 \mathrm{e}$ $35 \mathrm{~mm}$, respectivamente). O pequeno aumento e a dificuldade de obtenção de resíduos vegetais fazem com que esta cobertura morta não seja prática promissora na região.
\end{abstract}

Palavras-chave: balanço hídrico, feijão, milho, déficit hídrico, cobertura morta

\section{WATER BUDGET IN A SOIL WITH FOOD CROPS IN THE SEMI-ARID REGION OF NORTHEAST BRAZIL}

\begin{abstract}
Considering that water is a limiting factor and there are few studies on water balance in the semi-arid Northeastern Brazil, an experiment was established at Coxixola, PB, Brazil, with four treatments (corn and beans crops, bare soil and soil covered with mulch), in plots of $7.7 \times 10 \mathrm{~m}$ and plant spacing of $1.1 \times 1.0 \mathrm{~m}$. Rainfall, evaporation (Class A tank) and soil water (neutron probe) were monitored. Low $(212 \mathrm{~mm})$ and irregular rainfall resulted in water deficit towards the final period of the experiment and was responsible for the low water use efficiencies and low productivity of corn (grain and total biomass, 282 and $1141 \mathrm{~kg} \mathrm{ha}^{-1}$ ) and beans ( 166 and $558 \mathrm{~kg} \mathrm{ha}^{-1}$ ). Bare soil and mulch had similar results, with high water losses, 1.57 and $1.48 \mathrm{~mm} \mathrm{day}^{-1}$, respectively, and only small increases in stored soil water (28 and $35 \mathrm{~mm}$, respectively) were observed at the end of experiment. Mulching does not seem to be a promising practice in the area due to this small increase and the difficulty in obtaining plant residues.
\end{abstract}

Key words: water balance, beans, corn, water deficit, mulch

Recebido em 12/08/1999, Protocolo 089/99

${ }_{1}^{1}$ Professor Doutor do Departamento de Energia Nuclear, UFPE, Av. Prof. Luís Freire 1000, Recife, PE, CEP 50740 - 540.

Fone: (0xx81) 271 8252,Fax: (0xx81) 271 8250. E-mails: acda@npd.ufpe.br; esampaio@npd.ufpe.br; salcedo@npd.ufpe.br

${ }^{2}$ Professor Mestre do Departamento de Energia Nuclear, UFPE. E-mail: ado@npd.ufpe.br 


\section{INTRODUÇÃO}

O Nordeste do Brasil compreende uma área de 1,5 milhão de $\mathrm{km}^{2}$ e é a região mais pobre do país. Os problemas socioeconômicos são mais acentuados na área semi-árida, que se estende por aproximadamente um milhão de $\mathrm{km}^{2}$ (SUDENE 1997). A agricultura de subsistência (geralmente milho e feijão) é a atividade econômica mais importante nesta região e a disponibilidade de água é o principal obstáculo para a produção agrícola. A área inclui uma grande variedade de sistemas agrícolas, resultantes de diferentes solos, topografias e padrões de chuva.

No semi-árido nordestino, pela sua própria definição, a água é o fator mais limitante à obtenção de elevadas produtividades agrícolas, de forma regular, ao longo dos anos; entretanto, outra característica regional é a extrema variabilidade das condições climáticas (Reddy, 1983) fazendo com que, em alguns anos, o suprimento de água às plantas seja suficiente para atingirem altas produtividades, enquanto em outros anos pode levar à perda total das colheitas.

As culturas de subsistência são normalmente estabelecidas em baixios (várzeas), com semeadura após as primeiras chuvas; muitas vezes, esta semeadura é perdida quando as plântulas morrem, por falta de água, devido à distribuição irregular da chuva e à ausência de água armazenada no perfil do solo.

Apesar da importância da disponibilidade hídrica na região semi-árida, são poucas as pesquisas sobre balanço hídrico do solo e a maior parte foi feita com irrigação. Sob condições irrigadas, também foram medidos a evapotranspiração de milho, em Barreiras, na Bahia (Oliveira et al., 1993) e os balanços hídricos de solos cultivados com feijão de corda em Pentecoste, no Ceará (Saunders et al., 1981) e com capim buffel em Sumé, na Paraíba (Dantas Neto et al., 1996); sem irrigação, ainda em Pentecoste, foi medido o balanço hídrico de solo cultivado com feijão de corda (Saunders et al., 1985) e determinada a relação entre evaporação e umidade do solo mantido sem vegetação (Aguiar et al., 1998).

A área costeira nordestina, mais úmida, tem sido mais estudada e servido para o desenvolvimento de modelos de dinâmica de água no solo (Bernardo et al., 1996; Carneiro \& de Jong, 1986) aplicáveis também ao semi-árido. Em outras regiões brasileiras encontra-se maior número de trabalhos publicados sobre balanço hídrico de solos cultivados com milho (Caseiro et al., 1997) e feijão (Rodrigues et al., 1998), principais culturas do semi-árido nordestino.

Para contribuir com a disponibilização de informação sobre uso de água no semi-árido, realizou-se um experimento no município de Coxixola, PB, um dos locais de menor pluviosidade no Nordeste brasileiro, com o objetivo de se determinar o balanço hídrico de solo cultivado com milho ou feijão, em condições de sequeiro, e de solo nu ou com cobertura morta.

\section{MATERIAL E MÉTODOS}

O experimento de campo foi conduzido na Fazenda Quixaba, no município de Coxixola, latitude: $7^{\circ} 41^{\prime}$ S e longitude: $36^{\circ} 38^{\prime} \mathrm{W}$ ) na microrregião dos Cariris Velhos, PB. A precipitação pluviométrica local para o ano de 1996 foi de 436,5 mm, com temperatura média de $28^{\circ} \mathrm{C}$ e evapotranspiração potencial em torno de $2000 \mathrm{~mm}$. O experimento foi instalado em uma área de várzea com solo aluvial profundo, com as seguintes características químicas determinadas utilizando as metodologias recomendadas pela EMBRAPA (1997): pH em água 6,2, P e K, extraídos pelo método de Mehlich, 21,7 e $278 \mathrm{mg} \mathrm{kg}^{-1}$, respectivamente.

Realizou-se a caracterização física do solo da área experimental mediante análise de granulometria e da retenção de água a 33 e $1500 \mathrm{kPa}$ (capacidade de campo e ponto de murcha permanente, respectivamente) em amostras coletadas a cada $15 \mathrm{~cm}$, até a profundidade de $90 \mathrm{~cm}$ (Tabela 1). Na análise granulométrica as frações de argila e silte foram determinadas por sedimentação, após dispersão com hexametafosfato de sódio, utilizando-se o método da pipeta e a fração areia foi separada por peneiramento (Loveland \& Whalley, 1991). A retenção de água no solo foi determinada utilizando-se "panela de pressão” com placa porosa (Reeve \& Carter, 1991).

Tabela 1. Características físicas de camadas do solo da área experimental

\begin{tabular}{|c|c|c|c|c|c|}
\hline \multirow{3}{*}{$\begin{array}{l}\text { Profundidade } \\
\mathrm{cm}\end{array}$} & \multicolumn{3}{|c|}{ Granulometria } & \multicolumn{2}{|c|}{ Retenção de água } \\
\hline & Areia & Silte & Argila & $33 \mathrm{kPa}$ & $1500 \mathrm{kPa}$ \\
\hline & & $\%$ & & $\mathrm{~cm}$ & $\mathrm{~cm}^{-3}$ \\
\hline $0-15$ & 87,3 & 5,1 & 7,6 & 12,3 & 6,0 \\
\hline $15-30$ & 83,1 & 5,4 & 11,5 & 15,9 & 7,8 \\
\hline $30-45$ & 75,1 & 8,1 & 16,8 & 24,2 & 11,2 \\
\hline $45-60$ & 73,2 & 8,6 & 18,2 & 22,0 & 10,4 \\
\hline $60-75$ & 71,9 & 8,9 & 19,2 & 22,5 & 9,9 \\
\hline $75-90$ & 72,8 & 7,1 & 20,1 & 22,8 & 11,4 \\
\hline
\end{tabular}

Foram estabelecidos quatro tratamentos, com quatro repetições, em blocos casualizados: 1) plantio com milho; 2) plantio com feijão; 3) sem plantio e solo mantido nu; 4) sem plantio e solo com cobertura morta, constituída de restos culturais, mato proveniente de limpa e pontas de rama de plantas de caatinga, totalizando $27,6 \mathrm{Mg} \mathrm{ha}^{-1}$ de matéria seca. Cada repetição constou de uma parcela de 7,7 x 10,0 m com fileiras espaçadas $1,1 \mathrm{~m}$ e covas espaçadas $1,0 \mathrm{~m}$. As covas foram abertas com enxada, a uma profundidade de aproximadamente $15 \mathrm{~cm}$, a semeadura foi realizada após as primeiras chuvas, em 13 de março, e a colheita do experimento em 22 de julho de 1996. Para avaliação da produção consideraram-se, como áreas úteis, as três fileiras centrais de covas de cada parcela.

$\mathrm{Na}$ parte central de cada parcela foi instalado um tubo de acesso, em alumínio, para a sonda de nêutrons. Utilizou-se, para as determinações de umidade, uma sonda TROXLER série 3300, que contém uma fonte radioativa de Am-Be de atividade de $3,7.10^{8} \mathrm{~Bq}(10 \mathrm{mCi})$. As medições foram realizadas diariamente a cada $10 \mathrm{~cm}$, até a profundidade de $1 \mathrm{~m}$; também diariamente foram medidas a precipitação pluviométrica e a evaporação em tanque "classe A".

A curva de calibração da sonda de nêutrons foi obtida a partir da relação entre a umidade volumétrica e a contagem normalizada da sonda $(\mathrm{CN})$, isto é, a razão entre o número de contagens, $\mathrm{N}$, obtido no solo e o número de contagens, $\mathrm{N}_{\mathrm{p}}$, obtido para o mesmo intervalo de tempo, num moderador padrão (água) isto é, $\mathrm{CN}=\mathrm{N} / \mathrm{N}_{\mathrm{p}}$. As umidades volumétricas foram obtidas a partir de determinações gravimétricas de três amostras cilíndricas de 7,0 $\mathrm{cm}$ de diâmetro e $8,3 \mathrm{~cm}$ de altura, coletadas com amostrador de Ulhand, próximas ao tubo de acesso, logo 
após serem realizadas as medidas com a sonda de nêutrons. A reta de calibração obtida foi:

$$
\theta=0,003+0,772 \mathrm{CN}, \text { com } \mathrm{R}^{2}=0,98
$$

A partir da equação do balanço hídrico (Libardi, 1995) a evapotranspiração real foi obtida entre duas datas, por:

$$
\mathrm{ETR}=\mathrm{P}+\mathrm{I} \pm \mathrm{D} \pm \mathrm{R}-\Delta \mathrm{A}
$$

em que $\mathrm{P}$ e $\Delta \mathrm{A}$ representam a precipitação pluviométrica e a variação de armazenamento no perfil de solo $(0-1 \mathrm{~m})$. Considerou-se que não houve escoamento superficial de água, $\mathrm{R}$, dada a topografia plana da área, e que as perdas em água além do sistema radicular, ou drenagem profunda, $\mathrm{D}$, foram nulas.

O balanço hídrico para os quatro tratamentos foi efetuado considerando-se o perfil de solo estudado $(0-1 \mathrm{~m})$; para isto, os 120 dias estudados, de 23 de março a 20 de julho de 1996, foram divididos em nove subperíodos (seis de 13 dias e três de 14 dias).

Uma forma de se avaliar o estado hídrico a que está submetida uma cultura é através da relação entre a evapotranspiração real (ETR), e a evapotranspiração de referência (ET0), denominada coeficiente de cultura. Neste trabalho, a evapotranspiração de referência foi obtida pelo método do tanque de evaporação "Classe A", utilizando-se o valor 0,79 para o coeficiente do tanque (Freitas, 1994).

Os resultados foram submetidos a análise de variância, segundo um delineamento em blocos casualizados. Para análise da produção vegetal, foram comparados apenas os dados da colheita dos dois tratamentos cultivados. A análise do balanço hídrico foi feita como um arranjo fatorial 4 x 9 (tratamentos $\mathrm{x}$ subperíodos de cultivos).

\section{RESULTADOS E DISCUSSÃO}

A precipitação pluviométrica total, durante o experimento, foi de $212,5 \mathrm{~mm}$, com uma distribuição bastante irregular (Figura 1). Cerca de $81 \%$ da precipitação ocorreram nos quatro primeiros subperíodos, nos quais a distribuição da precipitação foi também irregular e dois episódios pluviais, um no segundo e outro no terceiro subperíodo, representaram cerca de $44 \%$ das suas precipitações. A evapotranspiração de referência totalizou 507,4 $\mathrm{mm}$, com média diária de 4,2 $\mathrm{mm}$. Um valor mais alto $\left(7,42 \mathrm{~mm} \mathrm{dia}^{-1}\right)$ foi encontrado no município de Sumé (Dantas Neto et al., 1996) vizinho a Coxixola, sendo a diferença originada, provavelmente, da diferença em época de medida; em Coxixola na época de chuva, a de maior umidade relativa do ar, maior nebulosidade e menor temperatura e, em Sumé, em época mais seca. Em Barreiras, na Bahia (Oliveira et al., 1993) os valores subiram de 3,37 a 6,3 $\mathrm{mm}$ dia $^{-1}$, do mês mais frio (junho) ao mais quente do experimento (setembro). Em Pentecoste, no Ceará, a média diária, de fevereiro a junho, foi de $3,67 \mathrm{~mm}$.

As variações de armazenamento de água no perfil do solo, para os quatro tratamentos, acompanharam as variações na precipitação, com diferenças significativas entre subperíodos (Figura 1). Também houve diferenças significativas entre tratamentos e na interação tratamento x subperíodos. Nos três
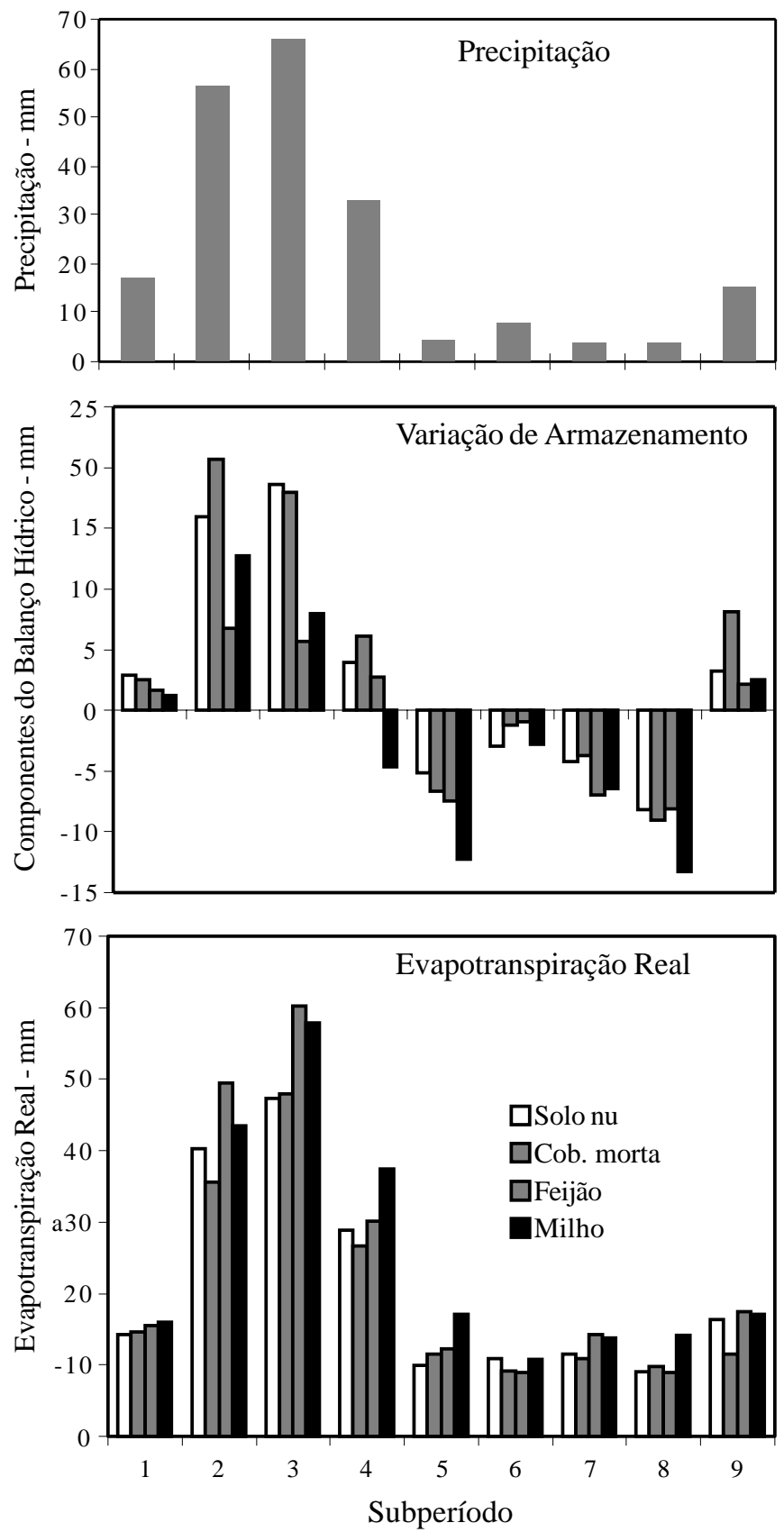

Figura 1. Componentes do balanço hídrico para os quatro tratamentos ao longo do ciclo de cultivo, dividido em subperíodos de 13-14 dias

primeiros subperíodos, ocorreu acumulação de água no solo dos quatro tratamentos, enquanto no quarto subperíodo o balanço ainda foi positivo para três dos tratamentos, mas negativo para o cultivo de milho. Esta maior perda de água pelo milho que pelo feijão, deve ter sido causada pelo seu crescimento mais rápido, com maior acúmulo de massa vegetal. Nos quatro subperíodos seguintes, de pouca chuva, houve perda de água em todos os tratamentos e, no último subperíodo, aconteceu nova acumulação de água, em todos os tratamentos, causada por chuvas tardias que, normalmente, não ocorrem na área, nesta época do ano. Para a acumulação nos tratamentos cultivados neste subperíodo final contribuiu sua baixa transpiração, como consequiência do secamento das plantas, já no final de seu ciclo e tendo passado pelo estresse dos subperíodos anteriores. 
Ao final do experimento haviam armazenados no perfil do solo 24,1 e 34,8 mm de lâmina de água a mais que no início do experimento, para os tratamentos solo nu e cobertura morta, respectivamente (Tabela 2 ). Este aumento deveu-se a evaporações totais menores que a precipitação. $O$ comportamento bastante semelhante entre os tratamentos solo nu e com cobertura morta pode ser explicado pelo fato de que, num solo nu, a camada superficial do solo funciona como um mulch, sendo tanto mais eficiente quanto mais condutor for o solo. O secamento da parte superficial de um solo, em condições naturais, passa por três fases, definidas por Idso et al. (1974): 1) a primeira fase corresponde à evaporação potencial, a partir de um solo úmido, e a evaporação é, então, controlada pelas condições climáticas; 2) a segunda fase refere-se a um estado mais seco na superfície e a taxa de evaporação é, em parte, controlada pelo solo, em função da sua capacidade de conduzir a água das camadas mais profundas para a superfície; 3) a terceira fase diz respeito a um estado muito seco na superfície e o regime de evaporação é controlado pelos mecanismos de transferência de vapor e de adsorção no seio da matriz sólida do solo. Um solo bastante condutor pode atingir a terceira fase em três ou quatro dias, enquanto um menos condutor pode passar mais de sete dias na primeira fase (Antonino, 1992).

Tabela 2. Balanço hídrico para os quatro tratamentos*, no período de 23/03 a 20/07

\begin{tabular}{|c|c|c|c|c|}
\hline \multirow{2}{*}{ Tratamento } & \multirow{2}{*}{$\begin{array}{c}\text { Precipitação } \\
\mathrm{mm}\end{array}$} & VLA & ETR & \multirow{2}{*}{$\begin{array}{c}\text { ETRd } \\
\mathrm{mm} \mathrm{dia}^{-1}\end{array}$} \\
\hline & & \multicolumn{2}{|c|}{$\mathrm{mm}$} & \\
\hline Solo nu & 212,53 & $24,11 \mathrm{a}$ & $184,42 b$ & $1,57 \mathrm{~b}$ \\
\hline Cob. morta & 212,53 & $34,81 \mathrm{a}$ & $177,71 b$ & $1,48 b$ \\
\hline Feijão & 212,53 & $-4,50 b$ & $217,02 \mathrm{a}$ & $1,81 \mathrm{a}$ \\
\hline Milho & 212,53 & $-15,04 b$ & $227,57 \mathrm{a}$ & $1,90 \mathrm{a}$ \\
\hline 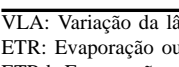 & & & & \\
\hline
\end{tabular}

Apesar das evaporações totais no solo nu e com cobertura morta terem sido inferiores à precipitação pluviométrica, elas foram relativamente altas, com médias diárias ao longo do experimento de 1,6e 1,5 mm (Tabela 2) e mesmo nos cinco últimos subperíodos ainda atingiram 0,78 e $0,85 \mathrm{~mm}$, respectivamente. A alta evaporação no tratamento com cobertura morta indica que esta técnica de conservação de água no solo não é uma prática promissora na agricultura do semi-árido nordestino, pois além da economia de água ter sido pequena, é difícil, arranjar material suficiente para uma boa massa de cobertura; os materiais usados poderiam ser restos culturais ou ramas da vegetação nativa mas, em geral, nesta área os restos culturais são usados na alimentação dos animais e, na época de plantio, a vegetação nativa está quase sem folhas (Sampaio \& Salcedo, 1997). Coberturas com plástico ou outros materiais sintéticos podem resultar numa economia hídrica maior mas não foram usadas porque não são usuais na região, dado o seu alto custo.

Enquanto nos tratamentos solo nu e cobertura morta houve aumentos nas quantidades de água armazenada no solo, nos tratamentos com cultivo houve reduções nas quantidades armazenadas e as diferenças absolutas foram significativas (Tabela 2). Para o feijão e o milho, os dados de evapotranspiração parecem bastante coerentes com os de precipitação (Tabela 2) e com as observações de campo, que indicaram baixas produtividades de grão e de palha (Tabela 3). A evapotranspiração média diária para o feijão foi de $1,81 \mathrm{~mm}$, que corresponde a $40 \%$ do valor obtido por Saunders et al. (1981) e a $48 \%$ do valor obtido por Bergamaschi et al. (1989) em condições de conforto hídrico, e a do milho foi de $1,90 \mathrm{~mm}$, perfazendo $55 \%$ do valor obtido por Reichardt et al. (1979) também nas mesmas condições. Em condições de sequeiro, no Ceará, a evapotranspiração média diária para o feijão foi de 3,18 mm (Saunders et al., 1985), quase o dobro da obtida neste trabalho; esta diferença talvez seja explicada pela quantidade e distribuição da precipitação pluviométrica durante os experimentos; no Ceará totalizou 459,4 mm, razoavelmente bem distribuídos no período experimental (março a julho) que correspondem a mais do dobro do total observado neste trabalho. A distribuição irregular em Coxixola, ligada ao baixo armazenamento no solo, resultou em grandes diferenças entre subperíodos: nos quatro primeiros, a evapotranspiração média diária para o feijão foi de 2,99 mm, pouco inferior à obtida no Ceará por Saunders et al. (1981) sob condições irrigadas ou de sequeiro (Saunders et al., 1985) enquanto nos cinco últimos foi de $0,90 \mathrm{~mm}$.

Tabela 3. Massas vegetais produzidas nas culturas de milho e feijão, índices de colheita (massa de grãos/massa total) e eficiência de uso de água (massa de água evapotranspirada/ massa vegetal produzida)*

\begin{tabular}{|c|c|c|c|c|c|}
\hline \multirow{2}{*}{ Cultivo } & \multicolumn{3}{|c|}{ Massas } & \multirow{2}{*}{$\begin{array}{c}\text { Índices } \\
\text { de Colheita }\end{array}$} & \multirow{2}{*}{$\begin{array}{c}\text { Eficiência de } \\
\text { Uso de Água } \\
\mathrm{Mg} \mathrm{Mg}^{-1}\end{array}$} \\
\hline & Grãos & $\begin{array}{l}\text { Planta } \\
\mathrm{kg} \mathrm{ha}^{-1}\end{array}$ & Total & & \\
\hline Milho & $282 a$ & $859 a$ & 1141a & 0,25 & $1994 b$ \\
\hline Feijão & $166 \mathrm{~b}$ & $392 b$ & $558 \mathrm{~b}$ & 0,30 & $3889 a$ \\
\hline
\end{tabular}

Até o quarto subperíodo, os valores da relação entre a evapotranspiração real e a evapotranspiração de referência (Figura 2) apresentaram boa concordância com os valores reportados na literatura (Oliveira et al., 1993; Doorenbos \& Kassam, 1994; Rodrigues et al., 1997). A partir do quinto subperíodo, as culturas começaram a sofrer restrição hídrica, responsável pela baixa produtividade final.

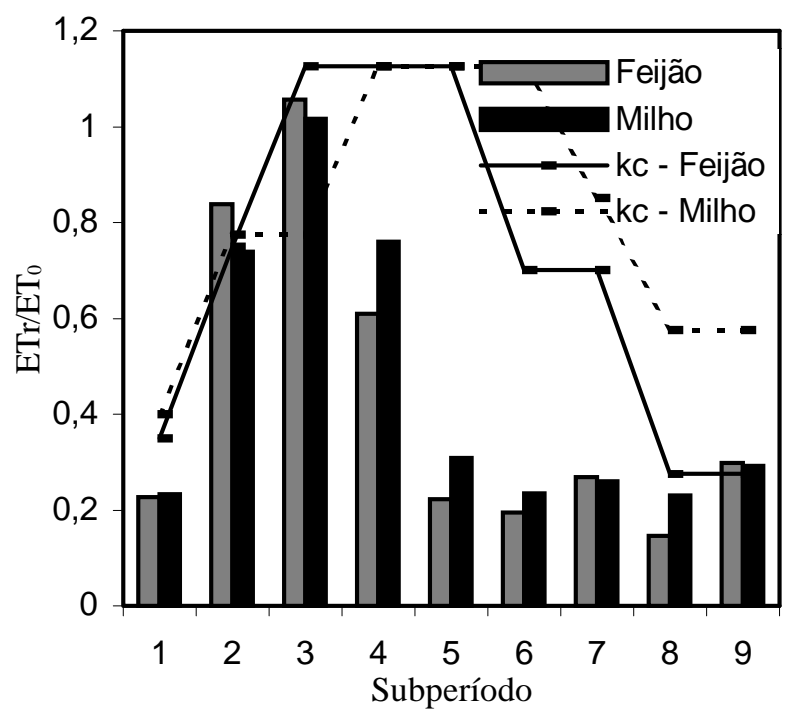

Figura 2. Relação entre a evapotranspiração real, ETR, e a evapotranspiração de referência, ET0, para o feijão e o milho e os valores médios do coeficiente de cultura, kc, reportados por Doorenbos \& Kassam (1994) 
A baixa produtividade final e a alta demanda evaporativa do ambiente explicam a baixa eficiência do uso de água, em ambas as culturas. Foram usados quase $2000 \mathrm{~kg}\left(2 \mathrm{~m}^{3}\right)$ de água para cada quilo de massa produzida, no caso do milho, e quase o dobro no caso do feijão (Tabela 3). A maior eficiência do milho pode ser creditada ao seu sistema fotossintético $\left(\mathrm{C}_{4}\right)$ com maior potencial de fixação de $\mathrm{CO}_{2}$ que o do feijão $\left(\mathrm{C}_{3}\right)$ mas ambas ficaram muito abaixo das eficiências obtidas em zonas com boas produtividades e que podem chegar a 200 e $500 \mathrm{~kg}$ de água por quilo de biomassa produzida, respectivamente (Boyer, 1996). A demanda evaporativa deve ter tido uma contribuição grande para essas baixas eficiências. Uma estimativa grosseira das contribuições pode ser feita deduzindo-se, das quantidades evapotranspiradas pelas culturas, as quantidades evaporadas nos tratamentos sem cultivo. Escolhendo-se o tratamento com cobertura morta para este cálculo (por ter menor evaporação e subestimar menos as transpirações) as perdas de água pelas culturas de milho e feijão seriam reduzidas para cerca de 50 e $40 \mathrm{~mm}$, respectivamente, e as eficiências seriam de cerca de 440 e $700 \mathrm{~kg}$ de água por kg de massa produzida. Os valores indicam que as perdas apenas por transpiração foram mais baixas que as por evaporação e para elas as eficiências de uso foram relativamente boas.

É relevante ressaltar que, apesar das baixas produtividades e eficiências de utilização de água, os índices de colheita não foram muito reduzidos, tendo a produção de grãos correspondido a 25 e $30 \%$ das produções totais de biomassa, no caso do milho e do feijão, respectivamente (Tabela 3) o que pode ser atribuído à adaptação das plantas à deficiência hídrica crescente, ao longo dos últimos subperíodos (Boyer, 1996). Mesmo assim, não deixa de surpreender, principalmente no caso do milho, em que as espigas tiveram tamanho reduzido, com poucas ultrapassando os $10 \mathrm{~cm}$ de comprimento, e com grãos muito espalhados. As plantas de milho também tiveram porte reduzido e poucas ultrapassaram 1,5 m de altura. A maior parte das vagens de feijão ultrapassou os $20 \mathrm{~cm}$ de comprimento, com bom enchimento de grãos, sendo a limitação na produção causada principalmente pelo baixo número de ramas e de vagens.

A não quantificação do termo de drenagem conduz aos seguintes comentários : 1) nos primeiros quatro subperíodos, pode ter ocorrido drenagem, principalmente nos segundo e terceiro subperíodos após os dois maiores episódios pluviais. A drenagem seria maior para os tratamentos solo nu e cobertura morta e faria com que os valores da evaporação real para esses tratamentos, sobretudo nos quatro primeiros subperíodos, fossem menores que os calculados sem drenagem; 2) no caso das parcelas cultivadas pode ter ocorrido certa compensação entre os valores de drenagem e ascensão capilar, nos quinto e sexto subperíodos. Esta suspeita se deve ao fato de, após o período chuvoso, o quinto e o sexto subperíodos terem apresentado baixos valores de evapotranspiração real, variação de armazenamento (Figura 1) e da relação ETR/ET0, principalmente no caso do feijão, com valores de cerca de 0,25 e 0,35 para o feijão e para o milho, respectivamente.

$\mathrm{Na}$ elaboração de balanços hídricos a maior dificuldade encontra-se na estimativa do termo de drenagem, obtido a partir da equação de Darcy. Existem sérias dificuldades na aplicação da equação de Darcy, devido a erros cometidos na estimativa do gradiente de potencial e, principalmente, na escolha do valor da condutividade hidráulica (Reichardt et al., 1979).

\section{CONCLUSÕES}

1. A pequena precipitação pluviométrica durante o experimento (212,5 mm) e sua distribuição irregular, concentrada nos primeiros dois meses, causaram restrição hídrica na fase final do experimento e foram responsáveis pelas baixas produtividades e baixas eficiências de uso de água das culturas.

2. As variações de armazenamento de água no perfil do solo acompanharam as variações na precipitação, mesmo nos tratamentos solo nu e com cobertura morta.

3. As grandes perdas de água nos tratamentos, solo nu e cobertura morta, ao longo de todo o período (ainda 0,78 e 0,85 $\mathrm{mm} \mathrm{dia}{ }^{-1}$, respectivamente, no último subperíodo) fizeram com que os aumentos nas lâminas de água armazenadas no perfil do solo, ao final do experimento, fossem apenas de 28 e $35 \mathrm{~mm}$, respectivamente.

4. Este pequeno efeito na conservação da água e a dificuldade de obtenção de material fazem com que a cobertura morta com resíduos vegetais não seja uma prática promissora na região.

\section{REFERÊNCIAS BIBLIOGRÁFICAS}

AGUIAR, J.V.; ANGELOCI, L.R.; FOLEGATTI, M.V. Relação entre a evaporação e a umidade de um solo Bruno não Cálcico sem vegetação, em condições semi-áridas. Revista Brasileira de Agrometeorologia, Santa Maria, v. 6, p.229-233, 1998.

ANTONINO, A.C.D. Modélisation des transferts de masse et de chaleur dans le système sol-plante-atmosphère. Influence de la variabilité spatiale des caractéristiques hydrodynamiques du sol. Grenoble: Universidade Joseph Fourier, 1992.195p. Tese Doutorado

BERNARDO, A. L .; ANTONINO, A.C.D.; CARNEIRO, C.J.G.; DALL'OLIO, A. Evapotranspiração da cultura do feijão caupi na Zona da Mata de Pernambuco. In: CONGRESSO GERAL DE ENERGIA NUCLEAR, 4, 1996, Rio de Janeiro. Anais... Rio de Janeiro, Associação Brasileira de Energia Nuclear, 1996. CD Rom.

BOYER, J.S. Advances in drought tolerance of plants. Advances in Agronomy, San Diego, v. 56, p.187-218, 1996.

CARNEIRO DA SILVA, C.; DE JONG, E. Comparison of two computer models for predicting soil water in a tropical monsoon climate. Agriculture and Forest Meteorology, v. 36, p. 249-262, 1986.

CASEIRO, F.T.; CAMPELO JÚNIOR, J.H.; PRIANTE FILHO, N. Evapotranspiração máxima e coeficiente de cultura do milho (Zea mays L.), no período seco, em Santo Antônio do Leverger - MT. Revista Brasileira de Agrometeorologia, Santa Maria, v. 5, p.177-182, 1997.

DANTAS NETO, J.; SOUZA, J.L.; MATOS, J.A.; GUERRA, H.O.C. Necessidades hídricas e eficiência de uso de água pelo capim buffel. Revista Brasileira de Agrometeorologia, Santa Maria, v. 4, p.25-28, 1996.

DOORENBOS, J.; KASSAM, A.H. Efeito da água no rendimento das culturas. Campina Grande: UFPB, 1994. 306p. Estudos FAO Irrigação e Drenagem 33

EMBRAPA. Manual de métodos de análise de solo. Brasília: EMBRAPA, 1997.212 p. 
FREITAS, J.A.G. Evapotranspirômetro de pesagem por sensor de carga hidráulica: Construção, calibração e testes. Recife: UFRPE, 1994. 79p. Dissertação Mestrado

IDSO, S.B.; REGINATO, R.J.; KIMBALL, B.A.; NAKAYAMA, F.S. The three stages of drying of field soil. Soil Science Society of America Proceedings. Madison. v.38, p.831-835, 1974.

LIBARDI, P.L. Dinâmica da água no solo. Piracicaba: Departamento de Física e Meteorologia - ESALQ/USP, 1995. 497p.

LOVELAND, P.J.; WHALLEY, R.W. Particle size analysis. In: SMITH K.A.; MULLINS C.E., ed. Soil analysis - Physical methods. New York: Marcel Dekker, Inc, 1991. cap. 6, p.271-328.

OLIVEIRA, F.A.; SILVA, J.J.S.; CAMPOS, T.G.S. Evapotranspiração e desenvolvimento radicular do milho irrigado. Pesquisa Agropecuária Brasileira, Brasília, v. 28, p.1407-1415, 1993.

REDDY, S.J. Climatic classification: The semiarid tropics and its environment - A review. Pesquisa Agropecuária Brasileira, Brasília, v. 18, p.823-847, 1983.

REEVE, M.J.; CARTER A.D. Water release characteristic. In : SMITH K.A.; MULLINS C.E. (ed.). Soil analysis - Physical methods. New York : Marcel Dekker, Inc, 1991. cap. 3, p.111-160.

REICHARDT, K.; LIBARDI, P.L.; SAUNDERS, L.C.U.; CADIMAZ, A. Dinâmica da água em solo cultivado com milho. Revista Brasileira de Ciência do Solo, Campinas, v. 3, p.1-5, 1979.
RODRIGUES, L.N.; MANTOVANI, E.C.; RAMOS, M.M.; SEDIYAMA, G.C. O modelo de Ritchie na determinação da evapotranspiração do feijoeiro (Phaseolus vulgaris L.) irrigado. Revista Ceres, Viçosa, v. 44, p.191-204, 1997.

RODRIGUES, L.N.; SEDIYAMA, G.C.; SOCCOL, O.J.; MANTOVANI, E.C. Modelo de Ritchie para determinações da transpiração do feijoeiro (Phaseolus vulgaris L.) e da evaporação direta da água do solo. Revista Brasileira de Agrometeorologia, Santa Maria, v. 6, p.179-185, 1998.

SAMPAIO, E.V.S.B.; SALCEDO, I.H. Diretrizes para o manejo sustentável dos solos brasileiros: Região semi-árida. In: CONGRESSO BRASILEIRO DE CIÊNCIA DO SOLO , 26, 1997, Rio de Janeiro. Anais dos Simpósios... Rio de Janeiro, Sociedade Brasileira de Ciência do Solo, 1997. CD-Rom.

SAUNDERS, L.C.U.; CASTRO, P.T.; BARBOSA C.E.; MATIAS, J. Dinâmica da água no solo com a cultura do feijão-de-corda (Vigna sinensis (L.) Savi) em aluvião eutrófico, Ceará. Ciência Agronômica, Fortaleza, v. 12, p.141-148, 1981.

SAUNDERS, L.C.U.; CASTRO, P.T.; BEZERRA F.M.L.; PEREIRA, A.L.C. Evapotranspiração atual da cultura do feijão-de-corda, (Vigna unguiculata L. Walp.) na microrregião-homogênea de Quixeramobim, Ceará. Ciência Agronômica, Fortaleza, v. 16, p.75-81, 1985.

SUDENE. Região Nordeste em números. Recife: SUDENE, 1997. $62 \mathrm{p}$. 